\title{
Health Implications of Marijuana Use: the Colorado Experience for Informed Decision-making in Israel
}

\author{
Richard Isralowitz ${ }^{1}$ (D) David A. Schwartz ${ }^{2}$. \\ Morton Mower ${ }^{3,4,5}$
}

Published online: 23 May 2018

(C) Springer Science+Business Media, LLC, part of Springer Nature 2018

Health factors associated with drug use such as cannabis show themselves in many ways that may affect quality of life. There is no shortage of people affected by such factors, nor is there any shortage of possible prevention and treatment interventions with ameliorative value. However, the uncommon is a multinational, multiethnic group of experts coming together to promote understanding and joint initiatives of mutual interest and importance.

Presenting the health implications of cannabis use by means of an edited special journal issue may be likened to craftsmanship. The task of collecting and organizing information from government and nongovernment authorities, researchers, educators, community service personnel, and others who have knowledge about health issues and drug use in different social and cultural contexts is no simple feat. To generate a final product that provides a coherent body of usable knowledge requires skill - it is a time consuming process that involves communication, cooperation, and coordination among the contributors and others involved.

This International Journal of Mental Health and Addiction special issue is an outcome of the "Health Implications of Marijuana Use: The Colorado Experience for Informed DecisionMaking in Israel" Symposium (January 6-14, 2018) hosted by Ben Gurion University of the Negev-Regional Alcohol and Drug Abuse Research (BGU RADAR) Center. The Symposium, a collaborative BGU and University of Colorado-Anschutz Medical Campus (UC) effort, was made possible by the generous support of Drs. Tobia and Morton Mower. In

Richard Isralowitz richard@bgu.ac.il

1 Regional Alcohol and Drug Abuse Research Center, Ben Gurion University of the Negev, Beer Sheva, Israel

2 Department of Medicine, School of Medicine, University of Colorado-Anschutz Medical Campus, Aurora, CO, USA

3 The Johns Hopkins University School of Medicine, Baltimore, MD, USA

4 Howard University College of Medicine, Washington, DC, USA

5 University of Colorado - Denver, Denver, CO, USA 
addition to generating usable knowledge about cannabis use legalization in Israel, the symposium promoted mutual interests and possible opportunities linking the academic institutions involved.

Cannabis is grown and traded almost everywhere; and, it is the most widely used "illegal" substance with about 183 million or $4 \%$ of the world's adult population consuming the drug at least once a year, more than all other illicit substances combined (UNODC World Drug Report 2017). It is a major growth industry for many countries and states creating new economic dynamics at individual, cooperative, conglomerate levels evolving in different ways. Cannabis use, over time, has been linked to political interests, racial bias, business and government profits, labor unionization, and other issues. It has been, and remains, a touchstone for local, state, national, and international debate.

The BGU RADAR Center that hosted the Symposium has considerable experience addressing drug issues. Approved by the US Substance Abuse and Mental Health Services Administration, the RADAR Center may be considered an outcome of the 1959 Middle East Narcotics Survey Mission of the United Nations. The UN Mission suggested ways to address illicit drugs including the substantial amounts of cannabis originating in the Middle East and transported to other parts of the world, in particular Europe and North America. From its beginning in 1996, the RADAR Center has been an "outpost" for drug abuse awareness in the Middle East region and has continued in this capacity longer than any similar nongovernment, academic institute in this part of the world. Recently, the US National Institute on Drug Abuse gave recognition to the BGU RADAR Center for its "contributions to scientific diplomacy through outstanding efforts in international collaborative research on drug abuse and addiction." Health and addiction experts, like those from the University of ColoradoAnschutz Medical Campus and other major academic institutions, have a significant role promoting BGU RADAR Center efforts.

Overall, the BGU-UC Symposium was an extraordinary event. The participants, with vast experience, included key Colorado and Israeli government personnel, BGU and UC decisionmakers and researchers, community-based service providers, and professionals from indigenous and immigrant communities.

We thank (in alphabetical order) all symposium and special issue participants. The participants from Colorado were; Ned Calonge; Mark Earnest, Morton Mower; Tobia Mower; David Schwartz; William Silvers; Larry Wolk; and Matthew Wynia. The Israeli participants were Ibrahim Abu Jaffer; Sarah Abu Kef; Yaniv Belhassen; Rivka Carmi; Orli GrinsteinCohen; Richard Isralowitz; Stuart Mirkin; Itay Pruginin; Alexander Reznik; Steve Rosen; Shahar Rubenstein; Orly Sarid; Vered Sarussi; Dorit Segal-Engelchin; Aharon Shabi; and Oren Wacht. Recognition is given to BGU RADAR Center team members for their exceptional organization efforts: Inbal Berman; Adi Dagan; and Mor Yehudai. Much appreciation is expressed to Masood Zangeneh, Editor, International Journal of Mental Health and Addiction (Springer International Publishing), for making this special issue possible and his unwavering support, over time, to Ben Gurion University, RADAR Center. 